\title{
Study on Stabilization Control System of Under Driven Ship
}

\author{
Yuting Xu, Zhuoqing Zhang \\ Nanchang Institute of Science \& Technology, Nanchang, Jiangxi, 330000
}

Keywords: Stabilization Control System, Under Driven Ship, Calculation Method

\begin{abstract}
In this paper, a conventional surface vessel is selected as the research object. It is pointed out that under specific control requirements, the system has an under-actuated characteristic and the problem of under-actuated ship stabilization control is studied. The dynamic mathematical model of ship motion is established and a simplified three-DOF under-actuated ship mathematical model is deduced. The non-complete characteristics of the system, the characteristic of balance point and the controllability of small time are analyzed.
\end{abstract}

\section{Introduction}

Ship motion control is an important research area of transportation engineering. It has been nearly a century old and its purpose is to improve the level of automation and intelligence of the ship so as to ensure the safety, economy and comfort of navigation. With the all-round prosperity and development of modern control theory and technology, especially the new theories and control algorithms of nonlinear control that have emerged in the past 20 years, it has injected vitality into the development of ship motion control science and also put forward new control problem. For a real control system, the system has several control outputs, we use several controllers to control them, in order to achieve the corresponding control objectives and performance requirements. However, if the original system needs to be controlled by several controllers, the same control effect can be achieved with only a few controllers. If feasible, the system cost and weight will be reduced. It is precisely from this idea that people are researching and developing new types of control systems. For a type of mechanical system, some degree of freedom is not installed with drive due to drive failure or special reasons, so that these degrees of freedom can not be controlled directly, and control can only be achieved through a combination of degrees of freedom. This type of mechanical system is called an under-driven mechanical system, ie, an under-actuated mechanical system is a type of mechanical system that controls the number of inputs less than the number of generalized coordinate variables or degrees of freedom of the system.

\section{Underwater driving surface watercraft mathematical model}

The mathematical model of ship movement is the most basic and important prerequisite for the study of ship stabilization control. It is the basis of ship control system analysis and the prerequisite for the design of ship stabilization controller. It is a necessary condition for experimental simulation. Therefore, the mathematical model of ship movement is directly related to the stabilization control effect of the whole system, which is very important and necessary for the stabilization control of under-powered ships. Mathematical models that describe ship motion are usually based on three aspects, namely, static dynamics, dynamic dynamics, and Newtonian mechanics. Dynamic dynamics refers to the situation where the system does variable speed motions under disturbances or self-motivation. Static dynamics refers to the situation where the system is moving at a constant speed or at the equilibrium point. However, what we need to build is the dynamic mathematical model of the under-actuated ship system because the under-powered ship under study is a response behavior under certain operating mode control or external disturbance. The mathematical models describing the movement of a ship are respectively kinetic and kinematic [1].

$\eta=[\mathrm{x}, \mathrm{y}, \varphi] \mathrm{T}$, where $\mathrm{x}, \mathrm{y}$ and $\varphi$ are the ship's position coordinates in the geodetic coordinate system and the heading of the ship respectively; $v=[u, v, r] T, C(v)$ is the Coriolis force and 
centripetal moment; $\mathrm{m}$ is the mass inertia matrix; $\mathrm{D}$ is the resistance matrix; $\mathrm{J}(\eta)$ is the hull coordinate $\mathrm{T}=[\tau 1, \tau 2, \tau 3] \mathrm{T}$, where $\tau 1, \tau 2, \tau 3$ are respectively the longitudinal thrust, lateral thrust and rotational moment of the ship under hull coordinate system; $\tau \mathrm{w}=[\tau \mathrm{w} 1, \tau \mathrm{w} 2, \tau \mathrm{w} 3] \mathrm{T}$, where $\tau \mathrm{w} 1, \tau \mathrm{w} 2$ and $\tau \mathrm{w} 2$ are the longitudinal and transverse disturbing forces and the disturbing moments in the direction of the rotating moment respectively[2].

The following assumptions are introduced according to the maneuverability of the ship: (1) Each element in the mass inertia matrix $\mathrm{m}$ and the resistance matrix $\mathrm{D}$ is a constant greater than zero and both are diagonal; and (2) the external disturbance is bounded.

\section{Controller design}

The backstepping method is actually a recursive design method (recursive design) from front to back, mainly the design problem of decomposing the feedback control from a higher order into a series of lower order systems. At each step, it changes the state coordinates, the adaptive adjustment function of uncertain parameters is related to the stabilization function of a known control system of Lyapunov function. The global adjustment or tracking of the system can be achieved by gradually correcting the deviation between the set trajectory and the actual trajectory. The backstepping method has brought a major breakthrough for the control of nonlinear systems. However, this method requires that the system structure must be a strict parameter feedback system or transformed into a nonlinear system of this type after transformation. Another major advantage of the backstepping method Is that it has the flexibility to avoid useful non-linearities in the cancellation system. The Back-stepping design can achieve global exponential stabilization and has good static and dynamic performance, which makes the Backstepping design method can often solve the system interference suppression, stabilization and tracking problems with less constraints. It can be seen from the figure that the under-driven ship system can be stabilized by a third-order chain system of the above system and the system $r=\tau r$. The system eventually needs to design $u 2$ and $\tau r$ to stabilize the new system to its origin. In order to make y3 converge exponentially, we choose the linear state feedback control $\mathrm{u} 1=-\mathrm{k} 3 \mathrm{y} 3, \mathrm{k} 3>0$ and the system becomes: $\mathrm{y} 1=-\mathrm{k} 3 \mathrm{y} 2 \mathrm{y} 3 \mathrm{y} 2=\mathrm{u} 2 \mathrm{y} 3$ $=-\mathrm{k} 3 \mathrm{y}\{3\}[3]$.

The problem is due to the fact that when the initial condition belongs to the set $\Omega=\{(y 1, y 2, y 3)$ $\in 3 \mathrm{y} 3(0) \neq 0, t \geq 0\}$, a time-varying feedback control law u2 The entire closed-loop system is bounded and exponentially converges to zero. The stabilization controller design process based on the backstepping method can be divided into three steps: Step 1: Construct the Lyapunov function $\mathrm{V} 1(\mathrm{y} 1)=12 \mathrm{y} 21$ and consider $\mathrm{y} 2$ as the virtual control input. In the set $\Omega=\{(\mathrm{y} 1, \mathrm{y} 2, \mathrm{y} 3)$ If $\mathrm{y} 2=\Psi 1$ $(\mathrm{y} 1, \mathrm{y} 3)$; and let $\Psi 1(\mathrm{y} 1, \mathrm{y} 3)=\mathrm{k} 1 \mathrm{y} 1 \mathrm{k} 3 \mathrm{y} 3, \mathrm{k} 1 \geq 0$ in $3 \mathrm{y} 3(0) \neq 0, \mathrm{t} \geq 0\}$. The derivative of $\mathrm{V} 1$ can be expressed as: $\mathrm{V} \bullet 1(\mathrm{y} 1)=\mathrm{y} 1 \quad \mathrm{y} 1=-\mathrm{k} 1 \mathrm{y} 21$

Step 2: Define a new variable: $1=\mathrm{y} 2-\Psi 1(\mathrm{y} 1, \mathrm{y} 3)$, which is used to describe the deviation between y2 and the virtual control input $\Psi 1(\mathrm{y} 1, \mathrm{y} 3)$. 代 $\mathrm{y} 1=-\mathrm{k} 3 \mathrm{y} 3(1+\Psi 1(\mathrm{y} 1, \mathrm{y} 3)) \cdot 1=\mathrm{u} 2+\mathrm{k} 1$ $(1+\Psi 1(\mathrm{y} 1, \mathrm{y} 3))-\mathrm{k} 1 \mathrm{y} 1 \mathrm{y} 3$ Substituting equation (23) into equation Let $\mathrm{V} \bullet 1(\mathrm{y} 1)=-\mathrm{k} 3 \mathrm{y} 1 \mathrm{y} 31$ k1y21 (24) Construct the Lyapunov function: (2) Let V1 (y1, y2) = $1221+\mathrm{V} 1$ ), We have: If we take the control law: $\mathrm{u} 2=-\mathrm{k} 1 \mathrm{y} 21+1(\mathrm{u} 2+\mathrm{k} 1(1+\Psi 1(\mathrm{y} 1, \mathrm{y} 3))-\mathrm{k} 1 \mathrm{y} 1 \mathrm{y} 3-\mathrm{k} 3 \mathrm{y} 1 \mathrm{y} 3)$ Substituting (27) into (26), let $\mathrm{k} 2 \geq 0$, we have: $\mathrm{V} \cdot 2(\mathrm{y} 1, \mathrm{y} 2)=-\mathrm{k} 1 \mathrm{y} 21-\mathrm{k} 221(\mathrm{k} 1+\mathrm{k} 2) \mathrm{y} 2+\mathrm{k} 3 \mathrm{y} 1 \mathrm{y} 3+\mathrm{k} 1(1+$ $\mathrm{k} 2 \mathrm{k} 3) \mathrm{y} 1 \mathrm{y} 3 \leq 0$

So when $t \rightarrow \infty$, y1 and $\square$ are bounded and tend to zero. Since y2 approaches k1y1/k3y3, in order to ensure the boundedness of y2 and to zero, we must ensure that $\mathrm{y} 1$ / y3 is bounded and tends to zero when $\mathrm{t} \rightarrow \infty$. Notice that $\mathrm{V} \cdot 1=-2 \mathrm{k} 1 \mathrm{~V} 1$, we can conclude that when $\mathrm{t} \rightarrow \infty, \mathrm{V} 1$ decays to zero at rate e- $2 \mathrm{k} 1 \mathrm{t}$, that is, y1 decays to zero at rate e- $\mathrm{k} 1 \mathrm{t}$, and since $\mathrm{y} 3$ decays at rate e- k3t decay to zero. Therefore, when $\mathrm{k} 1>\mathrm{k} 3$ and $\mathrm{y} 3(0) \neq 0$, it follows that $\mathrm{y} 1 / \mathrm{y} 3$ (ie, y2) is bounded and tends to zero at the rate of e- $(\mathrm{k} 1-\mathrm{k} 3) \mathrm{t}$.

Step 3: Define a new variable $2=\mathrm{u} 1-\Psi 2$ (y3), which is used to describe the relationship between $\mathrm{u} 1$ and the virtual control input $\Psi 2$ (y3) Between the deviation. $\bullet 2=\mathrm{u} 1-\Psi 2$ (y3) Construct the Lyapunov function V3 $(r)=12 \square 22$, and solve for $\mathrm{V} 3$ under the condition of the set $\Omega \cdot V \cdot 3(r)=2$ 
$(\tau r+k 3 r)$ satisfy the conditions of the parameters $k i, i=1,2,3, k 3$ is first selected to meet the dynamic changes y3 (ie z3); y1 / y3 rate selection depends on k1, from the previous knowledge we can see: And y1 have similar dynamic curve, you can take $\mathrm{k} 1=\mathrm{nk} 3$; parameter $\mathrm{k} 2$ selection will affect the dynamic response y2. To sum up, the system (1) through the construction of Lyapunov function, the resulting control law (27) and (31) enables the system to stabilize from any position to the origin of the inertial coordinate system. This shows that the stability of the new system shows that the original system is also stable.

Under-driven system refers to the system control input less than the degree of freedom of the system, which is characterized by fewer control inputs to control more degrees of freedom of movement. There are many reasons for this situation, which may be due to the inability of the system's implementing agencies to work properly or to reduce the number of implementing agencies for cost savings and weight savings. If the lateral propeller of the ship does not work properly for some reason, the degree of freedom in the lateral direction can not be controlled and it is necessary to control it by means of the forward propeller and the steering gear. An under-powered ship with a smaller number of drives to complete the mission, reducing the cost and weight of the system and improve operational efficiency, but also because of the reduction of control equipment to reduce the incidence of ship mechanical failure, the system more stable operation and easy to maintain. If the whole drive system in case of failure can not be normal operation, you can use under-drive control strategy, the use of the controller is still working to effectively control the system to increase equipment failure system reliability, under-drive control at the same time the ship fully driven The system provides a backup control technology. The under-actuated ship system belongs to non-complete system and does not satisfy the sufficient condition of Brocket's theorem. The steady state feedback control rate does not exist when the smooth continuous system stabilizes the system [4]. It also belongs to a special kind of nonlinear system, so it can not be solved by simple non-linear control method. As the stability control of nonholonomic systems can not be asymptotically stable with continuous smooth steady-state feedback, the linear control method is not ideal for under-drive stabilization. With the development of nonlinear control theory, many new methods are provided for the research of under-actuated ship stabilization control. For example, accurate linearization method, sliding mode variable structure control, adaptive control and so on. Under-actuated ship motion control mainly includes stabilization control, track-following control and formation control, while theoretical research on under-actuated ship attitude stabilization control began in the 1990s [5]. So far, the methods used in under-actuated ship stabilization control include: $\sigma$ transformation method, homogeneous method, feedback linearization, sliding mode variable structure control, back stepping method and intelligent control algorithm.

\section{Conclusions}

The research of this project focuses on the control of under-powered ships. At present, a considerable number of ships operating at sea are equipped only with helical forward thrusters and steering gears. For three-degree-of-freedom motion control of a horizontal plane, the ship has only two independent control inputs, and therefore, in essence, Drive System. The under-drive control of a ship can improve the economic performance of the ship and improve the safety of the ship's operation. Therefore, research on the motion control of under-driven ships has important theoretical significance and practical value.

\section{References}

[1] K Y Pettersen, O Egeland. Position and Attitude Control of an Under actuated Autonomous Underwater Vehicle[C]. Proceedings of the 35th Conference on Decision and Control, Kobe, Japan. December 1996: 987- 991.

[2] M Reyhanoglu, V S Arjan, N H Mc Clamroch. Dynamics and Control of a Class of under actuated Mechanical Systems[J]. IEEE Transactions on Automatic Control,1999,44(9): 121-122. 
[3] Frédéric Mazenc, Kristin Pettersen, Henk Nijmeijer. Global Uni-form Asymptotic Stabilization of an Underactuated Surface Vessel[J]. IEEE Transactions on Automatic Control, October 2002,47(10): 1759-1762.

[4] Jawhar Ghommam, Faical Mnif. Asymptotic Back Stepping Stabilization of an Under actuated Surface Vessel[J]. IEEE Transactionson control systems technology, November, 2006,14 (6): 1150-1157.

[5] K Y Pettersen, O Egeland. Position and Attitude Control of an Under actuated Autonomous Underwater Vehicle [J]. Proceedings of the 35th Conference on Decision and Control, Kobe, Japan. December 1996: 987- 991. 